\title{
Intervenção Coronária Percutânea no Diabetes Mellitus: Análise da Prática Médica Atual
}

\begin{abstract}
RESUMO
Os pacientes com diabetes e cardiopatia isquêmica são freqüentemente considerados para a realização de revascularização miocárdica com o objetivo de diminuição do risco de eventos cardiovasculares ou melhora da qualidade de vida. Na prática clínica, as decisões quanto à realização de cirurgia ou intervenção coronariana percutânea são freqüentemente difíceis, devido à gravidade dos casos, extensão da doença e presença de co-morbidades. Além disso, a maioria dos dados disponíveis na literatura origina-se da análise de subgrupos de ensaios clínicos delineados para estudos da população em geral, não para diabéticos. O objetivo deste estudo foi revisar a literatura quanto à intervenção coronária percutânea em pacientes com diabetes, além de mostrar dados mais recentes dos resultados deste procedimento no Serviço de Hemodinâmica do Instituto de Cardiologia do RS. (Arq Bras Endocrinol Metab 2007;51/2:327-333)
\end{abstract}

Descritores: Diabetes mellitus; Angioplastia coronariana percutânea; Revascularização miocárdica; Cardiopatia isquêmica

\section{ABSTRACT}

\section{Percutaneous Coronary Intervention in Diabetes Mellitus: An} Updated Analysis of Medical Practice.

Patients with diabetes and coronary artery disease are frequently considered for myocardial revascularization procedures, aiming at cardiovascular events risk reduction and a better quality of life. In clinical practice, decisions concerning surgery or percutaneous coronary intervention are frequently difficult, because of cases' severity, disease extension and co-morbidities association. Beyond that, the bulk of literature information was generated by subgroup analysis of randomized clinical trials, which were designed for the general population, not for diabetics. The aim of this study was to review literature on coronary percutaneous intervention in diabetic patients, and also to show recent data from the experience in this procedure at the Catheterization Laboratory of the Cardiology Institute of RS. (Arq Bras Endocrinol Metab 2007;51/2:327-333)

Keywords: Diabetes mellitus; Percutaneous coronary angioplasty; Myocardial revascularization; Coronary artery disease

$\bigcirc$ AUMENTO DO RISCO CARDIOVASCULAR associado ao diabetes mellitus (DM) está relacionado à própria doença $(1,2)$ e à presença de outros fatores de risco para doenças cardiovasculares $(1,3)$. Após o diagnóstico de doença cardiovascular, o paciente com diabetes também apresenta mais complicações e maior mortalidade do que aqueles indivíduos sem diabetes (4). O aumento da incidência de DM observado nos últimos anos (5) indica a necessidade de medidas preventivas e tratamento diferenciado para esses pacientes.

\section{atualização}

\author{
AleXANDRE QuADROS \\ FABIANE DIEMER \\ TATIANE LIMA \\ RICARDO ABDALLA \\ MATEUS VIZOTTO \\ Carlos A.M. Gottschall \\ BEATRIZ D'AGORD SCHAAN
}

Laboratório de Hemodinâmica, Instituto de Cardiologia do Rio Grande do Sul, Fundação Universitária de Cardiologia, Porto Alegre, RS.

Recebido em 31/12/06

Aceito em 04/01/07 
Os pacientes com DM e cardiopatia isquêmica são freqüentemente considerados para a realização de revascularização miocárdica $(\mathrm{RM})$ com o objetivo de diminuição do risco de eventos cardiovasculares ou melhora da qualidade de vida. Na prática clínica, considera-se que a RM é necessária na presença de sintomas como angina ou dispnéia, isquemia em testes de avaliação não-invasiva, comprometimento da função ventricular ou uma combinação destes fatores. No entanto, as decisões quanto à realização de cirurgia ou intervenção coronariana percutânea (ICP) são freqüentemente difíceis, devido à gravidade dos casos, extensão da doença e presença de co-morbidades. Além disso, a maioria dos dados disponíveis na literatura origina-se da análise de subgrupos de ensaios clínicos delineados para estudos da população em geral (6).

O objetivo do presente estudo foi revisar a literatura quanto à ICP em pacientes com DM, além de mostrar dados mais recentes dos resultados deste procedimento no Serviço de Hemodinâmica do Instituto de Cardiologia do RS.

\section{INTERVENÇÃO CORONARIANA PERCUTÂNEA (ICP)}

Ao analisarmos as questões relevantes em relação à ICP no paciente com DM, uma abordagem pragmática e com potencial aplicação para o clínico deve responder três perguntas: sem DM?

1. Quais as diferenças entre os pacientes com e

2. Quais as indicações de realização de ICP nos pacientes com DM?

3. Qual a ICP "ideal" no paciente com DM?

Nas seções seguintes, analisaremos as evidências científicas disponíveis em relação a estes três tópicos.

\section{Características e evolução clínica de pacientes com DM submetidos à ICP}

Os pacientes com DM submetidos à ICP apresentam risco aumentado de complicações e de reestenose, sendo que a presença de diabetes é um dos principais preditores de ambas as situações. A introdução dos stents como método preferencial de ICP foi acompanhada por uma redução de 25 a $30 \%$ nas taxas de reestenose angiográfica (7). Carrozza e cols. (8) foram os primeiros autores a descreverem uma maior freqüência de reestenose em indivíduos com DM comparados aos sem DM que usaram stents, achado que foi confirmado por diversos outros estudos $(9,10)$. A reestenose intra-stent diferencia-se daquela que ocorre após angioplastia por balão, caracte- rizando-se por proliferação neointimal e deposição de matriz extra-celular. O grau de proliferação neo-intimal é proporcional à lesão imposta e intensidade do processo inflamatório ocorrido, local e sistêmico (11). A proliferação excessiva das células musculares lisas da camada neointimal do vaso (12), diminuição da capacidade de regeneração das células endoteliais, alterações na produção local de vasodilatadores, anti-agregantes e substâncias anti-proliferativas (13), e sensibilidade exagerada das células musculares lisas do vaso às ações dos fatores próinflamatórios e trombóticos (14) são características do diabetes que contribuem para a ocorrência excessiva de reestenose nesses pacientes.

Em nosso meio, poucos estudos analisaram a evolução clínica de pacientes com DM tratados com implantes de stents coronarianos $(15,16)$. Recentemente, descrevemos a experiência do Instituto de Cardiologia de Porto Alegre com o implante de stents convencionais em 208 pacientes com DM que foram comparados com um grupo controle sem DM ( $\mathrm{n}=$ 685), sendo avaliados os resultados hospitalares e o seguimento clínico em um ano (12). Os pacientes com DM submetidos a implante de stents caracterizaram-se por ser um grupo de maior risco, mais idosos, mais fatores de risco para cardiopatia isquêmica e maior prevalência de revascularização prévia do miocárdio, além de comprometimento mais extenso das artérias coronárias. O sucesso do procedimento foi inferior nos pacientes com diabetes ( $88 \%$ vs. $92 \% ; \mathrm{p}=0,05)$, que também apresentaram maiores taxas de complicações hospitalares. As taxas de eventos cardiovasculares maiores (ECVM) em um ano foram maiores nos pacientes com diabetes do que nos controles (16,3\% vs. $9,3 \% ; \mathrm{p}=0,003)$, devido a maiores taxas de novas revascularizações $(12,3 \%$ vs. $8,0 \% ; p=0,06)$, infarto agudo do miocárdio (IAM, $3,0 \%$ vs. $1,4 \%, \mathrm{p}=0,05$ ) e óbitos $(5,4 \%$ vs. $2,5 \%$; $=0,03)$. Por análise multivariada, a presença de DM foi um preditor independente de ECVM em 1 ano (razão de chances: 2,00; intervalo de confiança: $1,25-3,24 ; p=0,004)$, bem como comprometimento de 3 vasos, diâmetro de referência do vaso-alvo e extensão da lesão.

Esses resultados são compatíveis com a tendência trombótica e aterogênica proporcionada pelo DM $(11,14)$, e que se traduz em maior risco de reestenose e reintervenção no vaso tratado $(17,18)$ e de complicações hospitalares (19). O risco aumentado de infarto agudo do miocárdio (IAM) e óbitos proporcionado pelo DM no seguimento em longo prazo se verifica tanto após o implante de stents, como relatado em nosso estudo (12) e noutros (20), mas também após a cirurgia de revascularização do mio- 
cárdio (21). Apesar de os stents farmacológicos diminuírem a necessidade de novas revascularizações nos pacientes com DM, este risco também é maior do que nos sem DM $(22,23)$.

\section{Indicações de ICP em pacientes com DM}

As indicações de ICP no paciente com diabetes são baseadas no mesmo racional aplicado para pacientes sem diabetes, que seria de proporcionar diminuição do risco de ECVM ou melhora da qualidade de vida quando comparado aos tratamentos alternativos, seja clínico ou cirúrgico. Neste contexto, o risco aumentado provocado pelos distúrbios metabólicos do diabetes desempenha um papel fundamental na escolha ou não de um método de revascularização miocárdica. Recentemente, foram publicados consensos atualizados para realização de ICP (24), e mesmo que este documento não enfoque especificamente os pacientes com DM, algumas orientações podem ser obtidas e que analisaremos a seguir. Em itálico encontramse as diretrizes resumidas, seguidas de comentários.

Em pacientes assintomáticos ou com angina estável, a ICP é uma indicação razoável naqueles com 1 ou mais lesões em 1 ou 2 coronárias, quando avaliada com alto indice de sucesso e baixo indice de complicações, sendo que o vaso tratado deve suprir uma grande área de miocárdio e com isquemia moderada à severa (classe II $A$, nivel de evidência $B$ ). Esta indicação reflete a preferência pela ICP em pacientes com doença arterial coronariana de 1 ou 2 vasos, mas também demonstra que sua realização não é obrigatória para todos os pacientes com estas características.

Em pacientes assintomáticos ou com angina estável, a efetividade da ICP em pacientes com lesões de 2 ou 3 vasos que têm comprometimento da $\mathrm{DA}$ proximale que são potenciais candidatos para cirurgia e que têm diabetes tratado ou disfunção ventricular esquerda não é bem estabelecida (classe II B, nípel de evidência B). Esta recomendação reflete a preferência pela indicação cirúrgica na maioria dos pacientes com doença arterial coronariana extensa, já que nos estudos randomizados a cirurgia diminuiu significativamente a incidência de ECVM no seguimento tardio, e em dois estudos observou-se diminuição da mortalidade (17,20-22, 25,26). Em pacientes com DM, a superioridade da cirurgia sobre a ICP deve-se ao impacto negativo no prognóstico decorrente da progressão da aterosclerose e o risco de IAM proporcionado pelos fatores já discutidos (27). Deste ponto de vista, a cirurgia substitui o vaso doente que tem placas vulneráveis passíveis de ruptura por um enxerto livre de lesões, enquanto a ICP trata somente um pequeno segmento da artéria coronária.
Por outro lado, deve-se ressaltar que os resultados dos ensaios randomizados podem não refletir a prática do chamado "mundo real", conforme demonstrado por um estudo de Detre e cols., no qual o registro de pacientes que recusaram a randomização prevista para o estudo BARI e que foram tratados de acordo com a conduta do médico assistente foi analisado (28). Nesta amostra de aproximadamente 350 pacientes com seguimento clínico de cinco anos, não houve diferença na incidência de óbitos e IAM entre pacientes com e sem DM, demonstrando que pacientes com DM podem ser tratados com ICP com segurança, desde que bem selecionados. Além disso, a maioria dos estudos disponíveis não incluiu o uso de stents farmacológicos, sendo que no ARTS II foi observada incidência significativamente menor de novos ECVM, o que é compatível com outros estudos $(21,29)$.

Em pacientes com sindromes coronarianas agudas sem supradesnivelamento do segmento ST, a estratégia invasiva precoce é indicada naqueles sem co-morbidades importantes e que apresentem lesões passíveis de ICP e algum dos seguintes fatores: 1) Isquemia recorrente; 2) Troponina positiva; 3) Depressão segmento ST; 4) Insuficiência Cardíaca; 5) Disfunção sistólica ventricular esquerda; 6) Instabilidade hemodinâmica e/ou taquicardia ventricular; 7) Cirurgia Cardiaca on ICP prévias (classe I, nível de evidência $A$ ). Esta recomendação reflete a superioridade do tratamento invasivo precoce sobre o tratamento clínico em pacientes com síndrome coronariana aguda sem supradesnivelamento do segmento ST de alto risco, conforme já demonstrado por diversos ensaios clínicos randomizados (30-32). Os pacientes com DM devem ser tratados com ICP precoce quando apresentarem algum dos marcadores de alto risco descritos.

As indicações de ICP ou cirurgia em pacientes com DM multiarteriais que se apresentam com síndrome coronariana aguda sem supradesnivelamento do segmento ST são semelhantes àquelas de pacientes com angina estável, conforme comentado anteriormente. Quanto às indicações de ICP nos pacientes com DM e IAM com supradesnivelamento do segmento ST, a conduta de decisão quanto à reperfusão primária deve ser a mesma dos pacientes sem DM.

\section{Intervenção coronariana percutânea ideal nos pacientes com DM}

A introdução dos stents farmacológicos foi um importante avanço da cardiologia intervencionista na última década, sendo que o seu benefício clínico é particularmente pronunciado nos pacientes com alto risco de reestenose, como pacientes com DM e aqueles com lesões longas ou 
em vasos finos (33-37). Em uma análise de 525 pacientes com DM incluídos em seis ensaios clínicos randomizados com acompanhamento de 9 meses, os stents farmacológicos diminuíram as taxas de revascularizações $(5,8 \%$ vs. $22,3 \% ; \mathrm{p}<0,0001)$ e de $\operatorname{ECVM}(8,9 \%$ vs. $24 \%$; $<<$ $0,0001)$ quando comparados aos stents convencionais.

Por outro lado, estudos recentes demonstraram que os stents farmacológicos também podem estar associados a aumento do risco de trombose e de mortalidade em longo prazo, o que poderia diminuir o benefício obtido pela diminuição da reestenose (38-43). Recentemente, o Food and Drug Administration (FDA) americano reuniu-se para uma re-análise extensa e meticulosa da segurança em longo prazo destes dispositivos (44). Para as indicações on-label, ou seja, aquelas analisadas nos ensaios clínicos randomizados e que constam na bula do produto, a conclusão do painel foi muito favorável. Nessas situações, o benefício dos stents farmacológicos claramente supera um pequeno risco de trombose tardia associada, e que não foi associado a aumento de mortalidade ou IAM. No entanto, quando os stents farmacológicos são utilizados em situações off-label, aquelas menos estudadas e geralmente mais complexas e graves, os membros do painel deixaram aberta a possibilidade de que as taxas de IAM e mortalidade possam ser aumentadas. É importante ressaltar que este aumento de eventos adversos também ocorre quanto os stents convencionais são usados em situações off-label, refletindo, portanto, a gravidade inerente destas situações clínicas. As situações onde o painel adverte para mais cautela com o uso de stents farmacológicos seriam bifurcações, overlap de stents, e vasos com trombo.

Um outro problema desses stents é sua relação custo-efetividade desfavorável em diversas situações, como pacientes com baixo risco de reestenose, pacientes tratados pelo sistema único de saúde ou quando é necessário o uso de múltiplos stents (45). Assim, se considerarmos as evidências disponíveis até o momento, e o fato de que a maioria dos pacientes com diabetes candidatos à revascularização percutânea apresentam aterosclerose difusa, com lesões longas e vasos de menor calibre, podemos concluir que os stents farmacológicos estão indicados em uma grande parcela dos procedimentos realizados nestes indivíduos. As prováveis exceções são aquelas com risco muito baixo de reestenose (vasos calibrosos e lesões curtas) ou risco alto de trombose tardia (indicações off-label ou impossibilidade do uso de tienopirídinicos a longo prazo).

Clopidogrel e aspirina devem ser empregados em todos os pacientes submetidos à ICP, iniciando a administração pelo menos seis horas antes da intervenção. Os inibidores da glicoproteína IIb/IIIa dimi- nuem as complicações agudas e o risco de IAM relacionado aos procedimentos de intervenção percutânea em pacientes com síndromes isquêmicas agudas. Nos pacientes com DM, estudos demonstram que essas drogas estão associadas com diminuição da mortalidade e da reestenose clínica por uma ação anti-inflamatória, além dos benefícios citados (46-48). Além disso, estudos recentes demonstram que, após o implante de stents farmacológicos, o principal preditor de trombose tardia é a suspensão do tienopiridínico, fazendo com que se considere o uso destas drogas por um tempo cada vez mais prolongado (> l ano) (38).

O controle glicêmico e a escolha da droga antihiperglicêmica ideal antes da ICP também têm sido ressaltados como fatores importantes na diminuição do risco de eventos cardiovasculares em pacientes com DM (49). Corpus e cols. compararam os desfechos clínicos e angiográficos de pacientes com diabetes que foram submetidos à angioplastia eletiva em ocasião de ótimo controle glicêmico versus controle glicêmico não-ótimo (50). Neste estudo, verificou-se que os pacientes do primeiro grupo apresentaram menores taxas de reestenose, reinternação por causa cardiológica e angina recorrente após 12 meses, sugerindo que o controle glicêmico ideal antes da angioplastia deve ser adotado.

Além das intervenções citadas, outras drogas representativas do tratamento clínico atual da doença aterosclerótica coronariana nos pacientes com DM também desempenham um papel importante no período periICP, como as estatinas, beta-bloqueadores e inibidores da enzima conversora da angiotensina. Um outro cuidado importante é o uso de hidratação parenteral antes e após o procedimento, o que se associa com diminuição do risco de insuficiência renal aguda, complicação mais freqüente no paciente com DM.

\section{CONCLUSÕES}

Os pacientes portadores de DM que são considerados para realização de ICP são mais idosos, apresentam doença arterial mais difusa e extensa e têm mais comorbidades do que aqueles sem a doença. Com base nas evidências científicas disponíveis, as indicações anatômicas de ICP em pacientes com DM mais aceitas são o comprometimento uniarterial ou biarterial sem DA proximal, comprometimento biarterial com DA proximal com características anatômicas favoráveis, comprometimento triarterial com características anatômicas favoráveis e sem disfunção VE ou revascularização incompleta em pacientes com alto risco cirúrgico por co-morbidades. Finalmente, a ICP ideal 
Tabela 1. Características e resultados dos principais estudos comparativos entre cirurgia e intervenção coronariana percutânea (ICP).

\begin{tabular}{lccccc}
\hline Estudo & ARTS & ERACI II & SoS & AWESOME & ARTS II \\
N & 1205 & 450 & 988 & 454 & 607 \\
Desfecho primário & ECVM & ECVM & RVA & Sobrevida & ECVM \\
& $(1$ ano $)$ & (30 dias) & & & $(1$ ano $)$ \\
Delineamento & ECR & ECR & ECR & ECR & Registro \\
& ICP vs. CRM & ICP vs. CRM & ICP vs. CRM & ICP vs. CRM & ICP \\
Diabetes, \% & 17 & 17 & 14 & 31 & 26 \\
Triarteriais, \% & 31 & 56 & 42 & 45 & 54 \\
Fração de ejeção, \% & 61 & ND & 57 & 45 & 60 \\
Inibidores da & NA & 28 & 8 & 11 & 33 \\
glicoproteína & & & & & \\
IIb/IIla, \% & & 22 vs. 19 & 22 vs. 12 & 52 vs. 39 & 10 \\
$\begin{array}{l}\text { ECVM em 1-3 anos, } \\
\text { (ICP vs. CRM, \%) }\end{array}$ & 26 vs. 12 & & & &
\end{tabular}

ECVM= Eventos Cardiovasculares Maiores; $I C P=$ Intervenção Coronariana Percutânea; $C R M=$ Cirurgia de Revascularização do Miocárdio; ECR= Ensaio Clínico Randomizado; RVA= Revascularização do Vaso-Alvo; ND= Não Disponível.

em pacientes com DM deve incluir stents farmacológicos provavelmente na maioria dos casos (com a provável exceção de lesões em vasos calibrosos ou lesões muito curtas, indicações off-label ou na impossibilidade do uso prolongado de tienopirídinicos). As drogas adjuntivas são aspirina, abciximab em pacientes com angina instável de alto risco e provavelmente naqueles com IAM, e clopidogrel $600 \mathrm{mg} \mathrm{em}$ pacientes com angina instável de baixo risco ou estável. Também é recomendada hidratação parenteral, estatinas, beta-bloqueadores, inibidores da enzima conversora da angiotensina e controle glicêmico rigoroso peri-procedimento de rotina.

\section{REFERÊNCIAS}

1. Stamler J, Vaccaro O, Neaton JD, Wentworth D. Diabetes, other risk factors, and 12-yr cardiovascular mortality for men screened in the Multiple Risk Factor Intervention Trial. Diabetes Care 1993;16(2):434-44.

2. Stratton IM, Adler Al, Neil HA, Matthews DR, Manley SE, Cull $\mathrm{CA}$, et al. Association of glycaemia with macrovascular and microvascular complications of type 2 diabetes (UKPDS 35): prospective observational study. BMJ 2000;321(7258):40512.

3. Schaan BD, Harzheim E, Gus I. [Cardiac risk profile in diabetes mellitus and impaired fasting glucose]. Rev Saúde Pública 2004;38(4):529-36.

4. Fox CS, Coady S, Sorlie PD, Levy D, Meigs JB, D'Agostino RB, Sr., et al. Trends in cardiovascular complications of diabetes. JAMA 2004:292(20):2495-9.

5. Fox CS, Pencina MJ, Meigs JB, Vasan RS, Levitzky YS, $\mathrm{D}^{\prime}$ Agostino RB, Sr. Trends in the incidence of type 2 diabetes mellitus from the 1970s to the 1990s: the Framingham Heart Study. Circulation 2006;113(25):2914-8.
6. Brooks RC, Detre KM. Clinical trials of revascularization therapy in diabetics. Curr Opin Cardiol 2000;15(4):287-92.

7. Serruys PW, de Jaegere P, Kiemeneij F, Macaya C, Rutsch W, Heyndrickx G, et al. A comparison of balloon-expandablestent implantation with balloon angioplasty in patients with coronary artery disease. Benestent Study Group. N Engl J Med 1994;331(8):489-95.

8. Carrozza JP, Jr., Kuntz RE, Fishman RF, Baim DS. Restenosis after arterial injury caused by coronary stenting in patients with diabetes mellitus. Ann Intern Med 1993;118(5):344-9.

9. Mehran R, Dangas GD, Kobayashi Y, Lansky AJ, Mintz GS, Aymong ED, et al. Short- and long-term results after multivessel stenting in diabetic patients. J Am Coll Cardiol 2004;43(8):1348-54.

10. Lau KW, Ding ZP, Johan A, Lim YL. Midterm angiographic outcome of single-vessel intracoronary stent placement in diabetic versus nondiabetic patients: a matched comparative study. Am Heart J 1998;136(1):150-5.

11. Scott NA. Restenosis following implantation of bare metal coronary stents: pathophysiology and pathways involved in the vascular response to injury. Adv Drug Deliv Rev 2006;58(3):358-76.

12. Schaan BD, Quadros A, Sarmento-Leite R, Gottschall CA. [Diabetes and coronary stent implantation: experience from a reference center in interventional cardiology]. Arq Bras Endocrinol Metab 2006;50(1):38-45.

13. Vanhoutte PM. Inducible nitric oxide synthase and vascular smooth muscle. Jpn J Pharmacol 1992;58(suppl 2):192P-9.

14. Kanzaki T, Shinomiya M, Ueda S, Morisaki N, Saito Y, Yoshida S. Enhanced arterial intimal thickening after balloon catheter injury in diabetic animals accompanied by PDGF beta-receptor overexpression of aortic media. Eur J Clin Invest 1994;24(6):377-81.

15. Mattos LA, Grines CL, Sousa JE, Sousa AG, Stone GW, Cox $D$, et al. One-year follow-up after primary coronary intervention for acute myocardial infarction in diabetic patients. A substudy of the STENT PAMI trial. Arq Bras Cardiol 2001;77(6):549-61.

16. Bayerl DM, Siqueira E, Moscoso I, Santos E, Maeda A, Bittencourt $\mathrm{O}$, et al. Coronary stent implantation in diabetic versus nondiabetic patients. Early and late outcomes. Arq Bras Cardiol 2000;75(5):365-8. 
17. Influence of diabetes on 5-year mortality and morbidity in a randomized trial comparing CABG and PTCA in patients with multivessel disease: the Bypass Angioplasty Revascularization Investigation (BARI). Circulation 1997;96(6):1761-9.

18. Mathew V, Gersh BJ, Williams BA, Laskey WK, Willerson JT, Tilbury RT, et al. Outcomes in patients with diabetes mellitus undergoing percutaneous coronary intervention in the current era: a report from the Prevention of REStenosis with Tranilast and its Outcomes (PRESTO) trial. Circulation 2004;109(4):476-80.

19. Van Belle E, Perie M, Braune D, Chmait A, Meurice T, Abolmaali $\mathrm{K}$, et al. Effects of coronary stenting on vessel patency and long-term clinical outcome after percutaneous coronary revascularization in diabetic patients. J Am Coll Cardiol 2002;40(3):410-7.

20. Legrand VM, Serruys PW, Unger F, van Hout BA, Vrolix MC, Fransen GM, et al. Three-year outcome after coronary stenting versus bypass surgery for the treatment of multivessel disease. Circulation 2004;109(9):1114-20.

21. Serruys PW, Ong AT, van Herwerden LA, Sousa JE, Jatene A, Bonnier JJ, et al. Five-year outcomes after coronary stenting versus bypass surgery for the treatment of multivessel disease: the final analysis of the Arterial Revascularization Therapies Study (ARTS) randomized trial. J Am Coll Cardiol 2005;46(4):575-81.

22. Rodriguez A, Bernardi V, Navia J, Baldi J, Grinfeld L, Martinez $J$, et al. Argentine Randomized Study: Coronary Angioplasty with Stenting versus Coronary Bypass Surgery in patients with Multiple-Vessel Disease (ERACI II): 30-day and one-year follow-up results. ERACI II Investigators. J Am Coll Cardiol 2001;37(1):51-8.

23. Liistro F, Fineschi M, Angioli P, Sinicropi G, Falsini G, Gori T, et al. Effectiveness and safety of sirolimus stent implantation for coronary in-stent restenosis: the TRUE (Tuscany Registry of Sirolimus for Unselected In-Stent Restenosis) Registry. J Am Coll Cardiol 2006;48(2):270-5.

24. Smith SC, Jr., Feldman TE, Hirshfeld JW, Jr., Jacobs AK, Kern MJ, King SB, 3rd, et al. ACC/AHA/SCAI 2005 Guideline Update for Percutaneous Coronary Intervention-summary article: a report of the American College of Cardiology/American Heart Association Task Force on Practice Guidelines (ACC/AHA/SCAI Writing Committee to Update the 2001 Guidelines for Percutaneous Coronary Intervention). Circulation 2006;113(1):156-75.

25. Morrison DA, Sethi G, Sacks J, Henderson W, Grover F, Sedlis $S$, et al. Percutaneous coronary intervention versus coronary artery bypass graft surgery for patients with medically refractory myocardial ischemia and risk factors for adverse outcomes with bypass: a multicenter, randomized trial. Investigators of the Department of Veterans Affairs Cooperative Study \#385, the Angina With Extremely Serious Operative Mortality Evaluation (AWESOME). J Am Coll Cardiol $2001 ; 38(1): 143-9$.

26. Coronary artery bypass surgery versus percutaneous coronary intervention with stent implantation in patients with multivessel coronary artery disease (the Stent or Surgery trial): a randomised controlled trial. Lancet 2002; 360 (9338):965-70.

27. Kuntz RE. Importance of considering atherosclerosis progression when choosing a coronary revascularization strategy: the diabetes-percutaneous transluminal coronary angioplasty dilemma. Circulation 1999;99(7):847-51.

28. Detre KM, Guo P, Holubkov R, Califf RM, Sopko G, Bach R, et al. Coronary revascularization in diabetic patients: a comparison of the randomized and observational components of the Aypass Angioplasty Revascularization Investigation (BARI). Circulation 1999;99(5):633-40.

29. Sabate M, Jimenez-Quevedo P, Angiolillo DJ, Gomez-Hospital JA, Alfonso F, Hernandez-Antolin R, et al. Randomized comparison of sirolimus-eluting stent versus standard stent for percutaneous coronary revascularization in diabetic patients: the diabetes and sirolimus-eluting stent (DIABETES) trial. Circulation 2005;112(14):2175-83.
30. Invasive compared with non-invasive treatment in unstable coronary-artery disease: FRISC II prospective randomised multicentre study. FRagmin and Fast Revascularisation during InStability in Coronary artery disease Investigators. Lancet 1999;354(9180):708-15.

31. Fox KA, Poole-Wilson PA, Henderson RA, Clayton TC, Chamberlain DA, Shaw TR, et al. Interventional versus conservative treatment for patients with unstable angina or non-ST-elevation myocardial infarction: the British Heart Foundation RITA 3 randomised trial. Randomized Intervention Trial of unstable Angina. Lancet 2002;360(9335):743-51.

32. Cannon CP, Weintraub WS, Demopoulos LA, Vicari R, Frey MJ, Lakkis $\mathrm{N}$, et al. Comparison of early invasive and conservative strategies in patients with unstable coronary syndromes treated with the glycoprotein IIb/IIla inhibitor tirofiban. N Engl J Med 2001;344(25):1879-87.

33. Sousa JE, Costa MA, Farb A, Abizaid A, Sousa A, Seixas AC, et al. Images in cardiovascular medicine. Vascular healing 4 years after the implantation of sirolimus-eluting stent in humans: a histopathological examination. Circulation 2004;110(1):e5-6.

34. Stone GW, Ellis SG, Cox DA, Hermiller J, O'Shaughnessy C, Mann JT, et al. A polymer-based, paclitaxel-eluting stent in patients with coronary artery disease. N Engl J Med 2004;350(3):221-31

35. Moses JW, Leon MB, Popma JJ, Fitzgerald PJ, Holmes DR, $\mathrm{O}^{\prime}$ Shaughnessy $\mathrm{C}$, et al. Sirolimus-eluting stents versus standard stents in patients with stenosis in a native coronary artery. N Engl J Med 2003;349(14):1315-23.

36. Gottschall CA, Quadros AS, Sarmento-Leite R. Predictive score for target vessel revascularization after bare metal coronary stenting. J Invasive Cardiol 2006;18(1):22-6.

37. Babapulle MN, Joseph L, Belisle P, Brophy JM, Eisenberg MJ. A hierarchical Bayesian meta-analysis of randomised clinical trials of drug-eluting stents. Lancet 2004;364(9434):583-91.

38. lakovou I, Schmidt T, Bonizzoni E, Ge L, Sangiorgi GM, Stankovic G, et al. Incidence, predictors, and outcome of thrombosis after successful implantation of drug-eluting stents. JAMA 2005;293(17):2126-30.

39. Ong AT, McFadden EP, Regar E, de Jaegere PP, van Domburg RT, Serruys PW. Late angiographic stent thrombosis (LAST) events with drug-eluting stents. J Am Coll Cardiol 2005;45(12):2088-92.

40. Nordmann AJ, Briel M, Bucher HC. Mortality in randomized controlled trials comparing drug-eluting vs. bare metal stents in coronary artery disease: a meta-analysis. Eur Heart J 2006;27(23):2784-814.

41. Spertus JA, Kettelkamp R, Vance C, Decker C, Jones PG, Rumsfeld JS, et al. Prevalence, predictors, and outcomes of premature discontinuation of thienopyridine therapy after drug-eluting stent placement: results from the PREMIER registry. Circulation 2006;113(24):2803-9.

42. Virmani R, Guagliumi G, Farb A, Musumeci G, Grieco N, Motta $T$, et al. Localized hypersensitivity and late coronary thrombosis secondary to a sirolimus-eluting stent: should we be cautious? Circulation 2004;109(6):701-5.

43. McFadden EP, Stabile E, Regar E, Cheneau E, Ong AT, Kinnaird $T$, et al. Late thrombosis in drug-eluting coronary stents after discontinuation of antiplatelet therapy. Lancet 2004;364(9444):1519-21.

44. Mayor S. Drug eluting stents are safe for licensed indications, FDA panel says. BMJ 2006;333(7581):1235.

45. Quadros AS, Gottshall CAM, Sarmento-Leite R. Custo-efetividade dos stents revestidos com drogas em vasos de grande calibre. Rev Bras Cardiol Invas 2006;14:306-13.

46. Mehilli J, Kastrati A, Schuhlen H, Dibra A, Dotzer F, von Beckerath $\mathrm{N}$, et al. Randomized clinical trial of abciximab in diabetic patients undergoing elective percutaneous coronary interventions after treatment with a high loading dose of clopidogrel. Circulation 2004;110(24):3627-35. 
47. Roffi M, Chew DP, Mukherjee D, Bhatt DL, White JA, Heeschen C, et al. Platelet glycoprotein Ilb/llla inhibitors reduce mortality in diabetic patients with non-ST-segmentelevation acute coronary syndromes. Circulation 2001;104(23):2767-71.

48. Bhatt DL, Marso SP, Lincoff AM, Wolski KE, Ellis SG, Topol EJ. Abciximab reduces mortality in diabetics following percutaneous coronary intervention. J Am Coll Cardiol 2000;35(4):922-8.

49. Sobel BE, Frye R, Detre KM. Burgeoning dilemmas in the management of diabetes and cardiovascular disease: rationale for the Bypass Angioplasty Revascularization Investigation 2 Diabetes (BARI 2D) Trial. Circulation 2003; 107(4): 636-42.

50. Corpus RA, George PB, House JA, Dixon SR, Ajluni SC, Devlin $\mathrm{WH}$, et al. Optimal glycemic control is associated with a lower rate of target vessel revascularization in treated type II diabetic patients undergoing elective percutaneous coronary intervention. J Am Coll Cardiol 2004;43(1):8-14.

Endereço para correspondência:

Beatriz D'Agord Schaan

Unidade de Pesquisa do IC/FUC

Av. Princesa Isabel 370

90620-001 Porto Alegre, RS

Fax: (51) 3230-3600 ramal 3777

E-mail:editoracao-pc@cardiologia.org.br/

beatrizschaan@terra.com.br 